\title{
Anti-Neutrophil Cytoplasmic Antibody (ANCA)-Negative Small Vessel Vasculitis: A Rare Cause of Pulmonary Renal Syndrome
}

\author{
Boudhayan Das Munshi, Sarbani Sengupta, Abhijeet Sharan, Sarmishtha Mukhopadhyay, \\ Bhaskar Ghosh, Angira Dasgupta and Rana Bhattacharyya
}

\begin{abstract}
Pulmonary renal syndrome (PRS) is characterized by both diffuse alveolar haemorrhage and glomerulonephritis as pathological features. Several immunologic and non-immunologic mechanisms including antineutrophil cytoplasmic antibody (ANCA)-positive vasculitis, anti-glomerular basement membrane disease, and systemic lupus erythematosus are commonly involved in the pathogenesis of the syndrome. We herein present a 60-year-old woman, non-smoker, who presented with fever, polyarthralgia, and the spreading of generalized purpuric rashes. The patient displayed rapid deterioration over the following two weeks marked by progressive declining renal function followed by haemoptysis. The patient was subsequently diagnosed with PRS, which was confirmed by the radiological evidence of alveolar haemorrhage and the histopathological evidence of pauci-immune glomerulonephritis. All immune markers including ANCA were negative. The patient was successfully treated with hemodialysis and immunosuppressive therapy. ANCA-negative vasculitis is a rare entity and even more rare as an etiology of PRS. An early diagnosis of this disease and its timely intervention is crucial.
\end{abstract}

Key words: pulmonary renal syndrome, anti-neutrophil cytoplasmic antibody (ANCA)-negative vasculitis

(Intern Med 54: 2759-2763, 2015)

(DOI: 10.2169/internalmedicine.54.4240)

\section{Introduction}

Pulmonary renal syndrome (PRS) is characterized by the combination of pathological features present in diffuse alveolar haemorrhage (DAH) and glomerulonephritis $(1,2)$. Underlying pulmonary pathology is small vessel vasculitis involving arterioles, venules, and alveolar capillaries. The renal pathology in PRS is predominantly in the form of necrotising glomerulonephritis (3). PRS patients are usually anti-neutrophil cytoplasmic antibody (ANCA)-positive; the detection of this antibody is pivotal in facilitating an early diagnosis of PRS. In contrast, PRS due to ANCA-negative systemic vasculitis is a rare entity. Here we report a case of ANCA-negative PRS with systemic features that have a unique presentation.

\section{Case Report}

A 60-year-old woman presented to our hospital with fever and polyarthralgia for the last 20 days, in addition to the swelling and presence of purpuric rashes on both legs for the last 14 days. Her fever was insidious in onset, low grade, and intermittent with no diurnal variation. It was accompanied by arthralgia of the large joints of her lower limbs followed by arthralgia of the large joints of her upper limbs. Within a few days, the small joints of all four of her limbs were also affected. Her fever was associated with a mild non-productive cough without any history of haemoptysis.

Five days after the patient's onset of fever, she developed multiple reddish non-pruritic elevated spots on her lower limbs, which were slightly larger than a pinhead and did not

Department of General Medicine, B R Singh Hospital and Center for Medical Education and Research, India Received for publication October 9, 2014; Accepted for publication February 23, 2015 Correspondence to Dr. Bhaskar Ghosh, drbghosh@gmail.com 


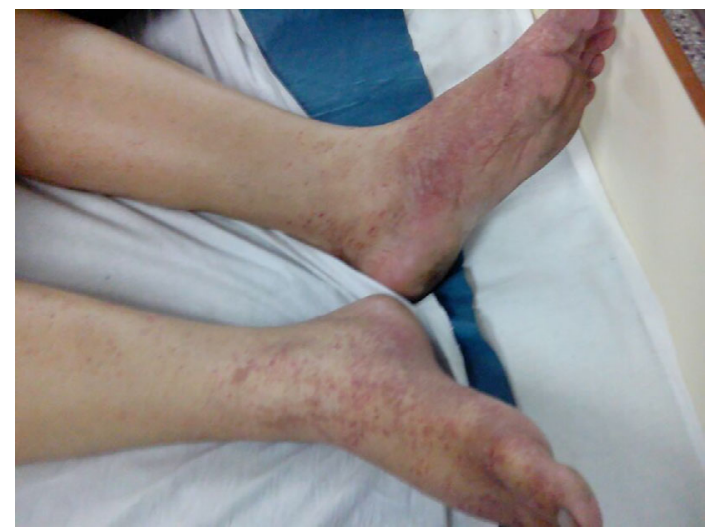

Figure 1. The vascultic skin lesions.

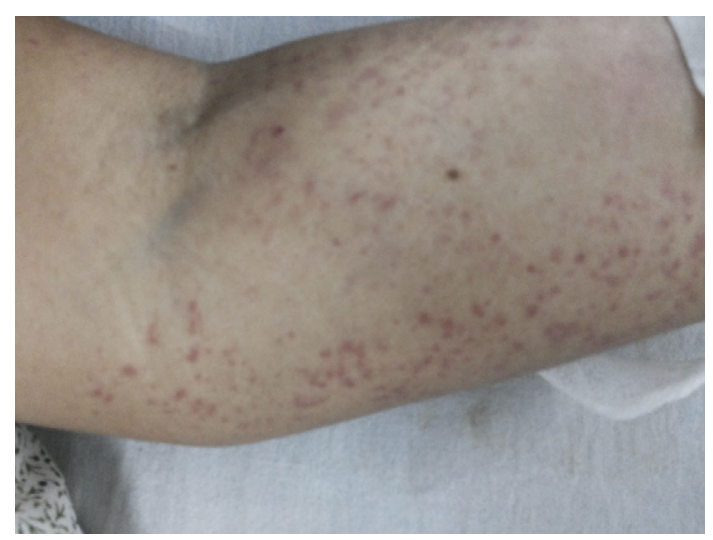

Figure 3. The vascultic skin lesions.

blanch on pressure. These purpuric spots gradually progressed to involve her entire body sparing the face. During the same time she noticed swelling of the feet, which gradually progressed to involve her legs up to the knees. It was associated with mild puffiness of the face but without any history of dysuria, haematuria, increased frequency of micturition or decreased urine output. The patient did not have any history of loss of appetite, night sweats, oral or genital ulcer, redness or dryness of eye, photosensitivity, sinusitis or epistaxis or colicky abdominal pain preceding or during the course of illness.

Her past medical history included only hypertension since 40 years of age for which she was on amlodipine $5 \mathrm{mg}$. She did not use any other regular medication, nor did she take any drug temporarily preceding the illness.

On clinical examination, the patient was an obese subject with a body mass index (BMI) of 30.3 (height 1.52 meter, weight $70 \mathrm{~kg}$ ), febrile, pale having resting tachycardia, and uncontrolled hypertension (B.P- 180/110 mm Hg) with bilateral pedal edema. She had palpable purpuric confluent skin rashes distributed all over the body and sparing the face, which were not photosensitive (Fig. 1-3). There were no cutaneous or mucosal ulcers, or subcutaneous nodules.

A musculoskeletal system examination revealed mild tenderness of all small and large joints without any effusion or deformity. The examination findings of respiratory, cardio-

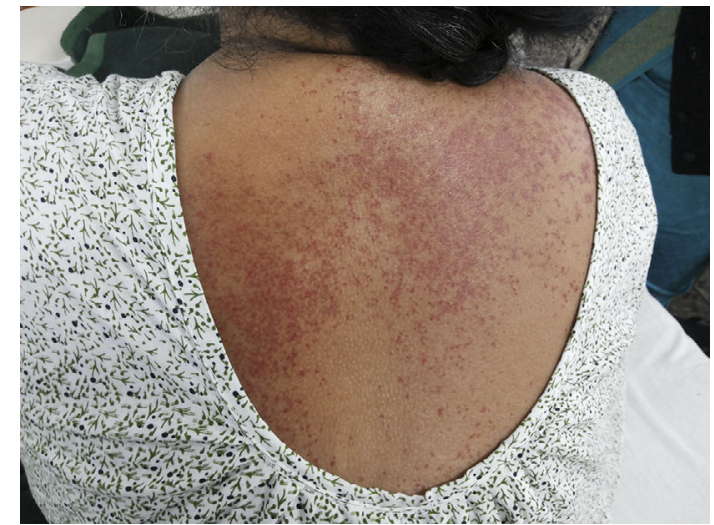

Figure 2. The vascultic skin lesions.

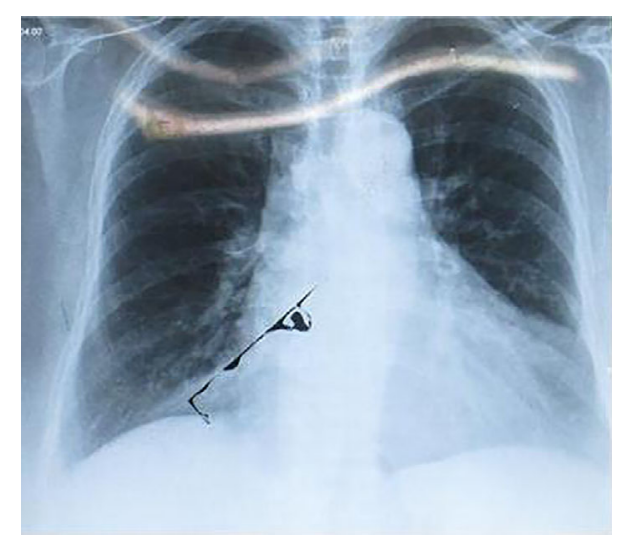

Figure 4. Chest X-ray on admission.

vascular, gastrointestinal, renal, and neurological systems were unremarkable.

Initial investigations revealed a haemoglobin of $8.2 \mathrm{~g} / \mathrm{dL}$, a total leukocyte count (TLC) of $11,600 / \mathrm{mm}^{3}$, and a differential leukocyte count (DLC) of the following: neutrophil $78 \%$, lymphocyte $18 \%$, eosinophil $3 \%$, monocyte $1 \%$, basophil $0 \%$. The patient's fasting blood glucose was $84 \mathrm{mg} / \mathrm{dL}$, urea was $41 \mathrm{mg} / \mathrm{dL}$ and creatinine was $1.6 \mathrm{mg} / \mathrm{dL}$. The results of the patient's initial chest X-ray were normal (Fig. 4). An ultrasound of the abdomen revealed normalsized kidneys with normal cortico-medullary differentiation, both kidneys being just hyperechoic. The urine examination showed 1-2 pus cells/high power field (HPF) with 20-30 red blood corpuscles (RBC)/HPF, 3+ albumin, few hyaline and granular casts, and a protein creatinine ratio of $744.95 \mathrm{mcg} /$ mg. The urine culture did not reveal growth of any organism. Other work-up performed for the fever was noncontributory.

The patient was started on antibiotics, anti-hypertensives, and other supportive management. During the course of her hospital stay, the patient developed a rapid worsening of renal profile (serum creatinine- $4.8 \mathrm{mg} / \mathrm{dL}$ ) however, her TLC normalized. Her urine, blood, and sputum cultures were all negative. Investigations to detect vasculitis (anti-nuclear antibody, anti-double stranded deoxyribonucleic acid, complements, extractable nuclear antigen profile, anti-phospholipid 


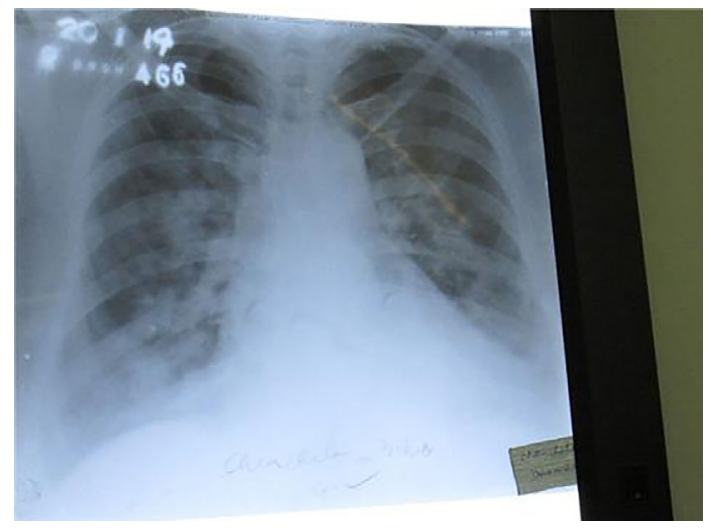

Figure 5. Chest X-Ray showing diffuse bilateral alveolar opacities.

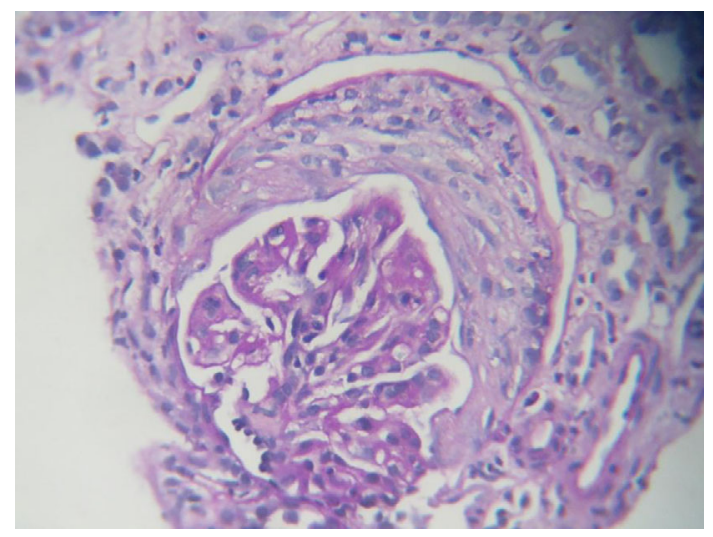

Figure 7. The renal biopsy showed 7 glomeruli, 4 of the glomeruli show presence of cellular crescent. There is increase in mesangial matrix and cellularity. Interstitium show moderate excess of mixed inflammatory cells. Tubules contain hyaline and granular cast. Blood vessels are unremarkable. Immunofluorescence assay (IgG, IgA, IgM, C3)-no glomerular deposits seen.

antibody test) yielded negative results. Both c-ANCA and pANCA were tested by indirect immunofluorescence assay followed by the detection of anti-myeloperoxidase (MPO) and proteinase-3 (PR-3) antigen by enzyme-linked immunosorbent assay (ELISA); the results were negative. The antiglomerular basement membrane (GBM) antibody test was also negative. A cryoglobulin qualitative test was also found to be negative. Serum protein electrophoresis did not show any $\mathrm{M}$ band. The patient's viral serology was screened for Dengue, Chikunguniya, human immune deficiency virus (HIV), and Hepatitis B and C and the results were negative.

During hospitalization, the patient underwent a renal and skin biopsy for an etiological diagnosis. Pending the biopsy reports, she was started on methylprednisolone $1 \mathrm{~g}$ I.V. for 3 days on the 12th day after admission. On the 15th day postadmission, the patient developed haemoptysis, in addition to respiratory distress with rapid fall in oxygen saturation. She also developed oliguria with a further rising trend of serum creatinine of $5.8 \mathrm{mg} / \mathrm{dL}$ for which she was put on haemo-

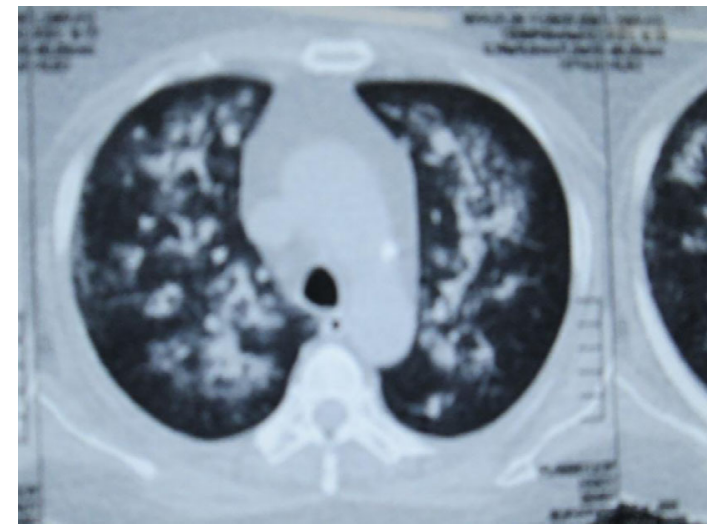

Figure 6. CT Thorax revealed fluffy and nodular peribrochovascular opacities in both lungs, mainly with perihilar distribution suggestive of alveolar haemorrhage.

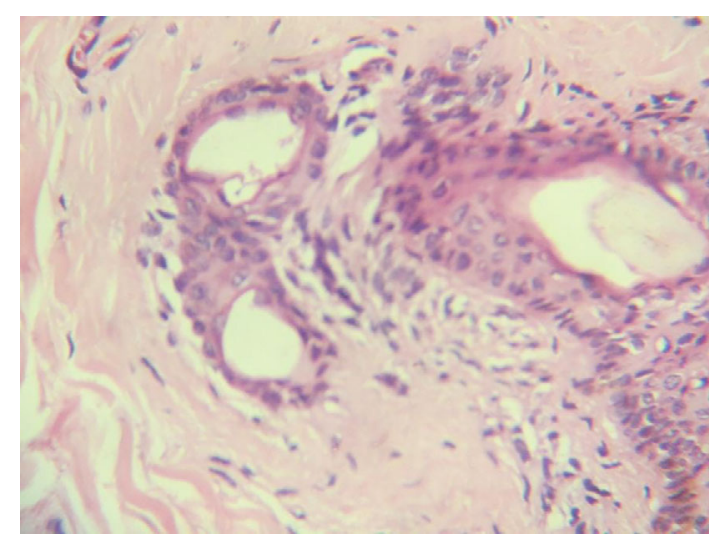

Figure 8. Skin biopsy showing non-specific vasculitis.

dialysis thrice weekly.

Chest X-rays performed this time presented diffuse bilateral alveolar opacities (Fig. 5). Computerised tomography (CT) of the thorax revealed fluffy and nodular peribrochovascular opacities in both lungs, mainly with perihilar distribution suggestive of alveolar haemorrhage (Fig. 6). This was confirmed by bronchoscopy in which the lavage fluid was haemorrhagic during sequential sampling.

The patient's renal biopsy (Fig. 7) showed 7 glomeruli; cellular crescent was present in 4 of the glomeruli. There was an increase in mesangial matrix and cellularity. The interstitium showed moderate excess of mixed inflammatory cells. The tubules contained hyaline and granular casts. The blood vessels were unremarkable. An immunofluorescence assay was performed to detect Immunoglobulin (Ig)G, IgA, IgM, and Complement 3 (C3) showed no glomerular deposits. A skin biopsy (Fig. 8) showed the presence of moderate excess of mixed inflammatory cells in the dermis without evidence of any granuloma. Histopathology was suggestive of focal leukocytoclastic vasculitis. Immunofluorescence for IgG, IgA, IgM, and C3 were negative. A diagnosis of ANCA-negative PRS was done.

The patient was treated with hemodialysis 3 times per week and given IV cyclophosphamide pulse therapy (12.5 
Table. Day Wise Representation of Symptoms, Serum Creatinine, and Treatment Done.

\begin{tabular}{|c|c|c|c|c|}
\hline DAY & SYMPTOMS & $\begin{array}{c}\text { SERUM } \\
\text { CREATININE }\end{array}$ & INVESTIGATION & TREATMENT \\
\hline On Admission & $\begin{array}{l}\text { Fever, Arthalgia, Rash, } \\
\text { Facial puffiness, Pedal } \\
\text { oedema, Pallor, } \\
\text { Hypertension }\end{array}$ & $1.6 \mathrm{mg} / \mathrm{dL}$ & $\begin{array}{l}\text { Routine blood, urine, } \\
\text { chest x-ray, } \\
\text { immunology, virology }\end{array}$ & $\begin{array}{l}\text { Antihypertensives, } \\
\text { Antibiotics, } \\
\text { Symptomatic treatment }\end{array}$ \\
\hline Day 8 & $\begin{array}{l}\text { Rapidly deteriotating } \\
\text { condition with oliguria }\end{array}$ & $4.8 \mathrm{mg} / \mathrm{dL}$ & Renal and skin biopsy & $\begin{array}{l}\text { Intravenous } \\
\text { Methylprednisolone } 1 \mathrm{~g}\end{array}$ \\
\hline Day 12 & & & & $\times 3$ days \\
\hline Day 15 & $\begin{array}{l}\text { Hemoptysis, Reduced } \\
\text { oxygen saturation, Fluid } \\
\text { overload, Oliguria }\end{array}$ & $5.8 \mathrm{mg} / \mathrm{dL}$ & Chest x-ray, CT thorax & $\begin{array}{l}\text { Hemodialysis } 3 / \text { week, } \\
\text { IV cyclophosphamaide } \\
\quad(12.5 \mathrm{mg} / \mathrm{kg}) \text { at } 3 \\
\text { weekly interval, Oral } \\
\text { prednisolone } 1 \mathrm{mg} / \mathrm{kg}\end{array}$ \\
\hline Day22 & $\begin{array}{l}\text { Hemoptysis subsided, } \\
\text { Oxygen saturation } \\
\text { normalised, Oliguria } \\
\text { persisted }\end{array}$ & $3.6 \mathrm{mg} / \mathrm{dL}$ & Follow up & $\begin{array}{l}\text { Hemodialysis } 3 / \text { week, } \\
\text { IV cyclophosphamaide } \\
\quad(12.5 \mathrm{mg} / \mathrm{kg}) \text { at } 3 \\
\text { weekly interval, Oral } \\
\text { prednisolone } 1 \mathrm{mg} / \mathrm{kg}\end{array}$ \\
\hline Day 29 & $\begin{array}{l}\text { Urine output increased, } \\
\text { No shortness of breath, } \\
\text { No hemoptysis }\end{array}$ & $1.1 \mathrm{mg} / \mathrm{dL}$ & Follow up & $\begin{array}{c}\text { Hemodialysis reduced } \\
\text { to } 1 / \text { week for } 3 \text { weeks, } \\
\text { then stopped. IV } \\
\text { cyclophosphamide } \\
\text { continued as before but } \\
\text { tapering of steroid } \\
\text { started }\end{array}$ \\
\hline 3 months & Symptom free & $1 \mathrm{mg} / \mathrm{dL}$ & Routine blood, urine & $\begin{array}{l}\text { Tapering dose of } \\
\text { prednisolone, } \\
\text { Azathioprin started, } \\
\text { later changed to } \\
\text { Mycophenolate } 500 \mathrm{mg} \\
\text { twice daily }\end{array}$ \\
\hline
\end{tabular}

$\mathrm{mg} / \mathrm{kg}$ ) every 3 weeks and oral prednisolone $1 \mathrm{mg} / \mathrm{kg}$ in tapering doses as induction therapy. Gradually, her haemoptysis subsided and her serum creatinine level decreased. After 3 months, the patient was put on oral azathioprine in the dose of $2 \mathrm{mg} / \mathrm{kg}$ as maintenance therapy. Later azathioprine was replaced by mycophenolate in the dose of $500 \mathrm{mg}$ twice daily as the patient could not tolerate azathioprine and till now the patient is doing well with prednisolone and mycophenolate. The effect of treatment on the symptoms and creatinine level corroborating with dialysis and immunosupression is shown in Table.

\section{Discussion}

PRS is characterized by the combination of diffuse alveolar haemorrhage and glomerulonephritis $(1,2)$. The three most common causes of PRS presenting to the physician are ANCA-positive small vessel vasculitis, anti-GBM disease (Goodpasture's disease), and systemic lupus erythematosus (SLE). The ANCA-positive vasculitides describe three major systemic syndromes namely granulomatosis with polyangiitis (Wegener's) (GPA), microscopic polyangiitis (MPA) and eosinophilic granulomatosis with polyangiitis (ChurgStrauss) (EGPA), which have subtle differences in clinical and pathological features. Immune-complex mediated PRS such as IgA vasculitis (Henoch-Schonlein) (IgAV), mixed cryoglobulinemia, and $\operatorname{IgA}$ nephropathy are rare but may present in a similar fashion (4). Drug-induced (propylthiouracil, penicillamine) PRS are also well described (5).

ANCA-negative PRS is a rare entity (6). Approximately $80-90 \%$ of the patients with PRS have one or more autoantibodies (6). Therefore, uncategorized PRS with negative serology for various antibodies, as in this case, is extremely rare. The lack of autoantibodies makes the diagnosis difficult and as in our patient, one has to depend on biopsies to make a correct diagnosis.

Our patient had a typical renal histopathological picture of cellular crescent associated with little to no glomerular staining for immunoglobulins that has been classically described in most cases of ANCA-negative pauci-immune rapidly progressive glomerulonephritis (6). The absence of deposits in immunofluorescent studies of renal biopsy excluded the possibility of post-infectious or immune complex origins of the disease. Additionally, we observed moderate interstitial infiltrates-mixed inflammatory cells, which is compatable with ANCA-negative (7) and ANCA-associated renal vasculitis (8). Skin biopsy results suggested features of vasculitis which is consistent with presence of systemic vasculitis. The CT thorax suggested pulmonary hemorrhage. Pulmonary, renal, and skin involvement along with myalgia, arthralgia, and fever (each organ involvement giving a score of $2 \times 4$ plus constitutional symptom score of 1) make disease extent index (DEI) score of 9 (9). As reported in litera- 
ture, ANCA-negative pauci-immune GN is part of a systemic vasculitic disease process (7). Additionally, the latter usually involves at least one extra-renal organ system as shown by the high DEI score of 9 in our patient, a score comparable with that of ANCA-positive vasculitis (9).

The unique development in our patient is her pulmonary haemorrhage. Only a small percentage of ANCA-negative pauce-immune vasulitis present with otolaryngological and pulmonary symptoms $(6,10)$ in contrast to ANCAassociated vasculitis where paranasal sinus and lung involvement are predominant, with a high prevalence of pulmonary haemorrhage $(40 \%)$ in MPO positive patients (11). The mechanisms leading to the occurrence of ANCA-negative vasculitis are not very clear. In ANCA-positive vasculitis, these auto-antibodies activate neutrophils either by direct $F$ (ab')2 binding to ANCA antigens on leukocyte surfaces, or Fc receptor engagement by ANCA immune complexes, leading to neutrophil and endothelial cell apoptosis and necrosis (12). However, in ANCA-negative vasculitis, the presence of neutrophil infiltration in pathological lesions occurs independently of circulating ANCAs, and may involve other unidentified autoantibodies or T-cell-dependent mechanisms (13). The fact that our patient responded well to immunosuppressive therapies further supports this theory.

When evaluating a patient with a possible vasculitis disorder with renal involvement, ANCA, anti-GBM and ANA assays currently play a critical role in the diagnosis and classification of vasculitic syndrome. A negative serology report may lead to a delay in the ultimate diagnosis as occurred in this case. A renal biopsy ultimately confirmed a diagnosis of ANCA-negative vasculitis with pauci-immune crescentic glomerulonephritis, which should always be contemplated whenever autoantibodies are negative in a clinically suspected case of PRS.

Patients who present with ANCA-negative vasculitis with pauci immune glomerulonephritis may have significant systemic involvement. Early diagnosis and early immunosuppressive therapy in the form of corticosteroids and cyclophosphamide bring about significant improvement in the prognosis. Further research is needed to elucidate the pathogenesis and focus on specific diagnostic markers for this group.

The authors state that they have no Conflict of Interest (COI).

\section{References}

1. Goodpasture EW. The significance of certain pulmonary lesions in relation to the aetiology of pneumonia. Am J Med Sci 158: 863$870,1919$.

2. Stanton MC, Tange JD. Goodpasture's syndrome (pulmonary haemorrhage associated with glomerulonephritis). Australas Ann Med 7: 132-144, 1958.

3. McCabe C, Jones Q, Nikolopoulou A, Wathen C, Luqmani R. Pulmonary-renal syndromes: An update for respiratory physicians. Respir Med 105: 1413-1421, 2011.

4. Jara LJ, Vera-Lastra O, Calleja MC. Pulmonary-renal vasculitic disorders: differential diagnosis and management. Curr Rheumatol Rep 5: 107-115, 2003.

5. Lock B, Eggert M, Cooper Jr JA. Infiltrative lung disease due to noncytotoxic agents. Clin Chest Med 1: 47-52, 2004.

6. Chen M, Feng Y, Wang SX, et al. Antineutrophil cytoplasmic autoantibody-negative pauci-immune crescentic glomerulonephritis. J Am Soc Nephrol 18: 599-605, 2007.

7. Eisenberger U, Fakhouri F, Vanhille P, et al. ANCA-negative pauci-immune renal vasculitis: histology and outcome. Nephrol Dial Transplant 20: 1392-1399, 2005.

8. Weidner S, Carl M, Riess R, Rupprecht HD. Histologicanalysis of renal leukocyte infiltration in antineutrophil cytoplasmic antibodyassociated vasculitis: importance of monocyte and neutrophil infiltration in tissue damage. Arthritis Rheum 50: 3651-3657, 2004.

9. De Groot K, Gross WL, Herlyn K, Reinhold-Keller E. Development and validation of a disease extent index for Wegener's granulomatosis. Clin Nephrol 55: 31-38, 2001.

10. Sampathkumar K, Ramakrishnan M, Sah AK, Gowtham S, Ajeshkumar RN. ANCA-negative pauci-immune glomerulonephritis with systemic involvement. Indian J Nephrol 20: 43-47, 2010.

11. Geffriaud-Ricouard C, Noel LH, Chauveau D, Houhou S, Grunfeld JP, Lesavre P. Clinical spectrum associated with ANCA of defined antigen specificities in 98 selected patients. Clin Nephrol 39: 125-136, 1993.

12. Jennette JC, Falk RJ. Pathogenesis of the vascular and glomerular damage in ANCA-positive vasculitis. Nephrol Dial Transplant $\mathbf{1 3}$ (Suppl 1): 16-20, 1998.

13. Cunningham MA, Huang XR, Dowling JP, Tipping PG, Holdsworth SR. Prominence of cell-mediated immunity effectors in 'pauci-immune' glomerulonephritis. J Am Soc Nephrol 10: 499-506, 1999.

(C) 2015 The Japanese Society of Internal Medicine http://www.naika.or.jp/imonline/index.html 